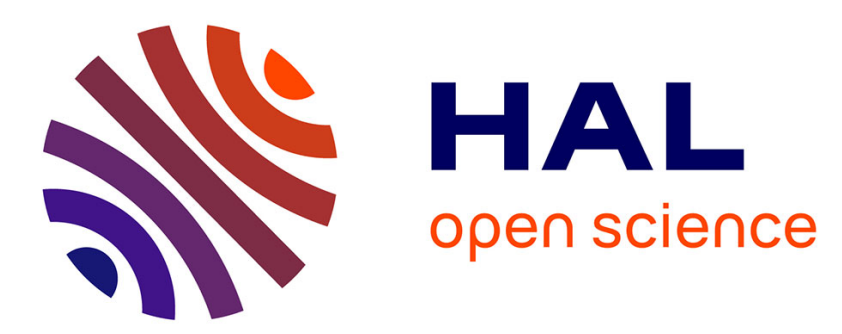

\title{
Decentralized Reasoning on a Network of Aligned Ontologies with Link Keys
}

\author{
Jérémy Lhez, Chan Le Duc, Thinh Dong, Myriam Lamolle
}

\section{To cite this version:}

Jérémy Lhez, Chan Le Duc, Thinh Dong, Myriam Lamolle. Decentralized Reasoning on a Network of Aligned Ontologies with Link Keys. JIAF 2019 - 13èmes Journées d'Intelligence Artificielle Fondamentale, Jul 2019, Toulouse, France. pp.1-10, 10.1007/978-3-030-30793-6_24 . hal-02302982

\section{HAL Id: hal-02302982 \\ https://hal.science/hal-02302982}

Submitted on 1 Oct 2019

HAL is a multi-disciplinary open access archive for the deposit and dissemination of scientific research documents, whether they are published or not. The documents may come from teaching and research institutions in France or abroad, or from public or private research centers.
L'archive ouverte pluridisciplinaire HAL, est destinée au dépôt et à la diffusion de documents scientifiques de niveau recherche, publiés ou non, émanant des établissements d'enseignement et de recherche français ou étrangers, des laboratoires publics ou privés. 


\title{
Decentralized Reasoning on a Network of Aligned Ontologies with Link Keys
}

\author{
Jérémy Lhez $^{1} \quad$ Chan Le Duc $^{1} \quad$ Thinh Dong $^{2} \quad$ Myriam Lamolle $^{1}$ \\ ${ }^{1}$ LIASD, IUT de Montreuil, Université Paris 8, France \\ ${ }^{2}$ Université de Danang, Vietnam \\ \{lhez, leduc, lamolle\}@iut.univ-paris8.fr, dnnthinh@kontum.udn.vn
}

\begin{abstract}
Résumé
Les clés de liage ont été récemment introduites pour la formalisation de données interconnectées entre des sources de données. Elles sont considérées comme un nouveau type de correspondances d'alignement d'ontologie. Nous proposons une procédure de raisonnement décentralisé sur un réseau d'ontologies avec alignements comprenant des clés de liage. Dans cet article, les ontologies incluses dans un tel réseau sont exprimées en logique de description $\mathcal{A} \mathcal{L} C$ alors que les alignements peuvent contenir des correspondences d'individu, de clé de liage et de concept. Ces dernières sont munies d'une sémantique affaiblie. L'aspect décentralisé de notre procédure est fondé sur un processus de propagation de connaissances à travers le réseau via les correspondances. Ce processus permet de réduire polynomialement le raisonnement global au raisonnement local.
\end{abstract}

\begin{abstract}
Link keys are recently introduced to formalize data interlinking between data sources. They are considered as a new kind of correspondences included in ontology alignments. We propose a procedure for reasoning in a decentralized manner on a network of ontologies with alignments containing link keys. In this paper, the ontologies involved in such a network are expressed in the logic $\mathcal{A} \mathcal{L} C$ while the alignments can contain concept, individual and link key correspondences equipped with a loose semantics. The decentralized aspect of our procedure is based on a process of knowledge propagation through the network via correspondences. This process allows to reduce polynomially global reasoning to local reasoning.
\end{abstract}

\section{Introduction}

Reasoning on a network of aligned ontologies has been investigated in different contexts where the semantics given to correspondences differs from one to another. To be able to develop a procedure for reasoning on a network of aligned ontologies, it is needed to equip the correspondences of the alignment with a semantics compatible with those defined in the ontologies. A simple approach to this issue consists in considering the correspondences as logical axioms expressed in the ontology language and merging all involved ontologies and the alignments into a unique ontology. In this case, the reasoning problem on such a network of aligned ontologies can be expressed as the following usual entailment :

$$
\bigcup_{1 \leq i \leq n} O_{i} \cup \bigcup_{1 \leq i<j \leq n} A_{i j} \vDash \alpha
$$

where $O_{i}$ is an $\mathcal{A} \mathcal{L} C$ ontology, $A_{i j}$ is an alignment between $O_{i}$ and $O_{j}$, and $\alpha 1$ is a link key or a concept assertion/axiom.

This approach is characterized by the following two main aspects : (i) the correspondences of the alignments are semantically handled as ontology assertion/axioms, and (ii) reasoning is performed on the unique ontology in a centralized manner, i.e. all reasoning tasks are carried out on a single location with a reasoner. Such an approach is quite unexploitable in the context of the Web where numerous ontologies and alignments are located in different sites. There have been researches [4, 6, 3, 13, 14, 1], which aimed at distributing reasoning over several locations. However, these approaches usually lead to an exponential blow-up of message passing between local reasoners associated with different locations. The main reason for this exponential blow-up is due to the strong semantics of the correspondences involved in the alignments.

In this paper, we introduce a new semantic of correspondences which are weaker than the usual ones and propose a

1. Consistency of the network can be reduced to the entailment 1 , with $\alpha=\perp(x)$ 
procedure for reasoning on a network of aligned ontologies in a decentralized manner-that means-reasoning can be independently performed on different sites following a process of knowledge propagation through the network of the ontologies via the alignments with link keys. Usefulness of link keys in Semantic Web applications and the problem of reasoning with them in the centralized context have been investigated by Atencia and Gmati [2, 5].

To illustrate our settings, we consider the following example in which knowledge is modelled in description logics. This formalism is used to encode the semantics of web languages such as OWL2.

Example 1. Consider two ontologies, denoted $\mathrm{O}_{1}$ and $\mathrm{O}_{2}$, where $O_{1}$ describes a terminology used by conference organizers, and $\mathrm{O}_{2}$ stores information about researchers and conferences they have attended. In $O_{1}$, there are classes Participant, Presenter, DemoPaperPresenter; and a property present. In $\mathrm{O}_{2}$, we can find classes Researcher, PhDStudent, Developer; and a property registerTo (i.e. someone registers to present a paper).

An alignment $A_{12}$ tells us that DemoPaperPresenter is simultaneously aligned with Researcher and Developer.

$$
\begin{aligned}
& \text { DemoPaperPresenter } \rightarrow \text { Researcher } \\
& \text { DemoPaperPresenter } \rightarrow \text { Developer }
\end{aligned}
$$

In addition, $A_{12}$ contains a link key which says that if a participant presents in the conference the same paper as that to which a researcher registers the conference then the participant and the researcher would be the same person.

$\{\langle$ present, registerTo $\rangle$ linkkey 〈Participant, Researcher $\rangle$

If we now add to $\mathrm{O}_{1}$ and $\mathrm{O}_{2}$ the following axioms/assertion

$$
\begin{array}{r}
O_{1}: \text { DemoPaperPresenter(Anna) } \\
O_{1}: \text { DemoPaperPresenter } \sqsubseteq \text { Participant } \\
O_{2}: \text { PhDStudent } \sqsubseteq \text { Researcher } \\
O_{2}: \text { Researcher } \sqsubseteq \neg \text { Developer }
\end{array}
$$

then a reasoner can find the entailment :

$$
\begin{aligned}
& O_{1} \cup O_{2} \cup A_{12} \vDash\{\langle\text { present, registerTo }\rangle \\
& \text { linkkey }\langle\text { DemoPaperPresenter, PhDStudent }\rangle
\end{aligned}
$$

This entailment holds because of the axioms (6), (7) and the link key (4). If we now interpret the correspondences (2) and (3) as subsumption in the standard semantics then the network $\mathrm{O}_{1} \cup \mathrm{O}_{2} \cup A_{12}$ is inconsistent because of the assertion/axiom (5) and (8). However, if we interpret these correspondences as a means for propagating concept unsatisfiability, i.e. unsatisfiability of the "subsumer" implies unsatisfiability of the "subsumee", then the network is consistent. In the following sections, we show that the weakened semantics corresponding to the latter interpretation of concept correspondences leads to a substantial change of the computational complexity of algorithms for reasoning.

In addition, the weakened semantics would not be really interesting for the correspondences (2) and (3). However, it would be more relevant for correspondences between ontologies of different nature. Given two ontologies about equipment and staff and a correspondence Computer $\rightarrow$ Developer between them. With this correspondence, the weakened semantics tells us that if there is no developer then there is no computer. The standard semantics is irrelevant in this case.

Based on the weakened semantics of alignments, we introduce in this paper the notion of consistency for a network of ontologies with alignments containing link keys (or an ontology network for short). Then, we propose an algorithm for checking consistency of an ontology network by reducing this task to checking consistency of each ontology which is polynomially extended. This consists in (i) propagating individual equalities of the form $a \approx b$ through all ontologies of the network via individual correspondences of the same form $a \approx b$, (ii) applying link keys in the alignments, which may lead to add new individual correspondences, (iii) propagating concept unsatisfiabilities through all ontologies of the network via concept correspondences of the form $C \rightarrow D$. We show that the complexity of the process of knowledge propagation is polynomial in the size of the network. In addition, we also prove that consistency of the ontologies and alignments extended by this process of knowledge propagation is equivalent to consistency of the network.

The remainder of the paper is organised as follows. Section 2 positions our work with respect to works on distributed reasoning in description logics. Section 3 describes the logic $\mathcal{A} \mathcal{L} C$ with individuals, alignments, a new semantics of alignments and inference services. Section 4 provides the algorithms for propagating individual equalities, applying link keys and propagating concept satisfiabilities. We also prove that reasoning on the ontology network is reducible to reasoning on each ontology extended by the algorithms, and this reduction is polynomial in the size of the ontology network. Section 5 presents examples of the use of the algorithms. Section 6 describes the architecture of Draon in which the algorithms are implemented in a decentralized manner. We also report some experimental results. Section 7 concludes the paper and presents future work.

\section{Related Work}

In the literature, there have been several reasoning approaches which either (i) merge all ontologies and alignments into a unique ontology and perform reasoning 
over that unique ontology, or (ii) use a distributed semantics such as DDL (Distributed Description Logics) [4], Econnection [6], IDDL (Integrated Distributed Description Logics) [13] , Package-based Description Logics [3] and design a distributed algorithm for reasoning. The second option consists in defining new formalisms which allow reasoning with multiple domains in a distributed way. The new semantics of these formalisms reconcile conflicts between ontologies, but they do not adequately formalize the quite common case of ontologies related with ontology alignments produced by third party ontology matchers. Indeed, these formalisms assert cross-ontology correspondences (bridge rules, links or imports) from one ontology's point of view, while often, such correspondences are expressed from a point of view that encompasses both aligned ontologies. Another issue of these non-standard semantics is that reasoners such as Drago [12], Pellet [10], an early version of Draon [8] using the distributed algorithms resulting from the corresponding semantics require an exponential number of message exchanges over network. This exponential blow-up results from exchanging model portions (the so-called distributed tableau) between modules of the reasoner located on different sites.

Recenty, Atencia and Gmati [2, 5] have proposed a tableau algorithm for reasoning in the centralized context on an $\mathcal{A} \mathcal{L} C$ ontology with link keys. They have showed that adding link keys to $\mathcal{A} \mathcal{L} C$ does not augment the complexity of the tableau algorithm.

\section{Preliminaries}

The syntax and semantics of the logic $\mathcal{A} \mathcal{L} C$ are defined below.

Definition 1 (Syntax of $\mathcal{A} \mathcal{L} C$ ). Let $\mathbf{C}, \mathbf{R}$ and $\mathbf{I}$ be nonempty sets of concept names, role names and individuals, respectively. The set of $\mathcal{A} \mathcal{L} C$-concepts (or simply concepts) is the smallest set such that every concept name in $\mathbf{C}$, $\mathrm{T}$ and $\perp$ are concepts, and if $C, D$ are concepts and $R$ is a role name in $\mathbf{R}$ then $C \sqcap D, C \sqcup D, \neg C, \forall R . C$ and $\exists R$. $C$ are concepts. A general concept inclusion (GCI) is an expression of the form $C \sqsubseteq D$ where $C, D$ are concepts. A terminology or TBox is a finite set of GCIs. An ABox assertion is an expression of the form $C(a), R(a, b), a \approx b$ or $a \neq b$ where $C$ is a concept, $R$ is a role name in $\mathbf{R}$ and $a, b$ are individuals in $\mathbf{I}$. An ABox is a finite set of ABox assertions. A pair $O=(\mathcal{A}, \mathcal{T})$, where $\mathcal{T}$ is a TBox and $\mathcal{A}$ is an $A B o x$, is called an $\mathcal{A} \mathcal{L} C$ ontology. We use $\operatorname{Voc}_{I}(O), \operatorname{Voc}_{C}(O)$ and $\operatorname{Voc}_{R}(O)$ to denote the sets of individuals, concept names and role names occurring in $O$.

Definition 2 (Semantics of $\mathcal{A} \mathcal{L} C$ ). An interpretation $\mathcal{I}=$ $\left(\Delta^{\mathcal{I}}, \cdot^{I}\right)$ is composed of a non-empty set $\Delta^{\mathcal{I}}$, called the domain of $\mathcal{I}$, and a valuation. ${ }^{I}$ which maps every concept name to a subset of $\Delta^{I}$, every role name to a subset of
$\Delta^{\mathcal{I}} \times \Delta^{\mathcal{I}}$ and each individual to an element of $\Delta^{\mathcal{I}}$. The valuation is extended to constructed concepts such that, for all concepts $C, D$ and role name $R$, the following is satisfied :

$$
\begin{aligned}
& \mathrm{\top}^{\mathcal{I}}=\Delta^{\mathcal{I}}, \perp^{I}=\emptyset,(\neg C)^{I}=\Delta^{I} \backslash C^{I} \\
& (C \sqcap D)^{I}=C^{I} \cap D^{I},(C \sqcup D)^{I}=C^{I} \cup D^{I} \\
& (\forall R . C)^{I}=\left\{x \in \Delta^{I} \mid \forall y .\langle x, y\rangle \in R^{I} \Rightarrow y \in C^{I}\right\} \\
& (\exists R . C)^{I}=\left\{x \in \Delta^{I} \mid \exists y .\langle x, y\rangle \in R^{I} \wedge y \in C^{I}\right\}
\end{aligned}
$$

An interpretation $I$ satisfies $a$ GCI $C \sqsubseteq D$, denoted by $\mathcal{I} \vDash C \sqsubseteq D$, if $C^{\mathcal{I}} \subseteq D^{\mathcal{I}}$. $\mathcal{I}$ is a model of a TBox $\mathcal{T}$ if $\mathcal{I}$ satisfies every GCI in $\mathcal{T}$. An interpretation $\mathcal{I}$ satisfies the ABox assertions : $C(a)$ if $a^{\mathcal{I}} \in C^{\mathcal{I}} ; R(a, b)$ if $\left\langle a^{\mathcal{I}}, b^{\mathcal{I}}\right\rangle \in$ $R^{I} ; a \approx b$ if $a^{\mathcal{I}}=b^{I} ; a \neq b$ if $a^{\mathcal{I}} \neq b^{\mathcal{I}}$. Given an ABox assertion $\alpha, \mathcal{I} \vDash \alpha$ denotes that $\mathcal{I}$ satisfies $\alpha$. $\mathcal{I}$ is a model of an ABox $\mathcal{A}$ if it satisfies every ABox assertion in $\mathcal{A}$. An interpretation $\mathcal{I}$ is a model of an $\mathcal{A} \mathcal{L} C$ ontology $O=(\mathcal{A}, \mathcal{T})$ if $\mathcal{I}$ is a model of $\mathcal{T}$ and $\mathcal{A}$. An ontology $O$ is consistent if there exists a model of $O$. $O$ entails alpha, written $O \models \alpha$, where $\alpha$ is a GCI or an assertion, if every model of $O$ satisfies $\alpha$.

We need notations and definitions that will be used in the paper. We use $|S|$ to denote the cardinality of a set $S$. Given an $\mathcal{A} \mathcal{L} C$ ontology $O=\langle\mathcal{A}, \mathcal{T}\rangle$, we denote by $\operatorname{sub}(O)=\operatorname{sub}(\mathcal{A}, \mathcal{T})$ the set of all sub-concepts occurring in $\mathcal{A}, \mathcal{T}$. The size of an ontology $O$ is denoted by $|O|=|\mathcal{A}|+|\mathcal{T}|$ where $|\mathcal{A}|$ is the size (number) of all assertions, $|\mathcal{T}|$ the size of all GCIs. It holds that $|\operatorname{sub}(O)|$ is polynomially bounded by $|O|$ since if a concept is represented by a string then a sub-concept is a substring.

To be able to define a network of aligned ontologies, we need alignments which represent semantic links between ontology entities such as individuals, concepts or roles.

Definition 3 (network of aligned ontologies). An $\mathcal{A} \mathcal{L} C$ network of aligned ontologies is a tuple $\left\langle\left\{O_{i}\right\}_{i=1}^{n},\left\{A_{i j}\right\}_{i, j=1, i \neq j}^{n}\right\rangle$ where $O_{i}$ is an $\mathcal{A} \mathcal{L} C$ ontology with $1 \leq i \leq n$, and $A_{i j}$ with $1 \leq i<j \leq n$ is an alignment containing correspondences of the following forms :

$-C \rightarrow D$ or $C \leftarrow D$ where $C \in \operatorname{sub}\left(O_{i}\right)$ and $D \in$ $\operatorname{sub}\left(O_{j}\right)$. Such a correspondence is called concept correspondence.

- $a \approx b(a \not b)$ where $a \in \operatorname{Voc}_{I}\left(O_{i}\right)$ and $b \in \operatorname{Voc}_{I}\left(O_{j}\right)$. Such a correspondence is called individual correspondence.

- a link key $\left\{\left\langle P_{k}, Q_{k}\right\rangle\right\}_{k=1}^{n}$ linkkey $\langle C, D\rangle$ where $P_{k} \in$ $\operatorname{Voc}_{R}\left(O_{i}\right), Q_{k} \in \operatorname{Voc}_{R}\left(O_{j}\right)$ for $1 \leq k \leq n, C \in \operatorname{sub}\left(O_{i}\right)$ and $D \in \operatorname{sub}\left(O_{j}\right)$. Such a correspondence is called link key correspondence.

The following definition formalizes the semantics of correspondences in an alignment so that it is compatible with that of ontologies. We retain the standard semantics for individual and link key correspondences while the semantics of concept correspondences is weakened. 
Definition 4 (semantics of alignments). An $\mathcal{A} \mathcal{L} C$ network of aligned ontologies is a tuple $\left\langle\left\{O_{i}\right\}_{i=1}^{n},\left\{A_{i j}\right\}_{i, j=1, i \neq j}^{n}\right\rangle$ where $O_{i}$ is an $\mathcal{A} \mathcal{L C}$ ontologies with $1 \leq i \leq n$, and $A_{i j}$ is an alignment with $1 \leq i<j \leq n$. Let $\mathcal{I}$ and $\mathcal{J}$ be models of $O_{i}$ and $O_{j}$ respectively.

- If $C \rightarrow D$ is in $A_{i j}$ then $D^{\mathcal{J}}=\emptyset$ implies $C^{\mathcal{I}}=\emptyset$.

- If $a \approx b$ is in $A_{i j}$ then $a^{\mathcal{I}}=a^{\mathcal{J}}$.

- If $a \not b$ is in $A_{i j}$ then $a^{\mathcal{I}} \neq a^{\mathcal{J}}$.

- If $\left\{\left\langle P_{k}, Q_{k}\right\rangle\right\}_{k=1}^{n}$ linkkey $\langle C, D\rangle$ is in $A_{i j}$ then $\left(a_{k}^{i}\right)^{\mathcal{I}}=$ $\left(a_{k}^{j}\right)^{\mathcal{J}},\left\langle a^{\mathcal{I}},\left(a_{k}^{i}\right)^{\mathcal{I}}\right\rangle \in P_{k}^{\mathcal{I}},\left\langle b^{\mathcal{J}},\left(a_{k}^{j}\right)^{\mathcal{J}}\right\rangle \in Q_{k}^{\mathcal{J}}$ for all $1 \leq k \leq n, a^{\mathcal{I}} \in C^{\mathcal{I}}, b^{\mathcal{T}} \in D^{\mathcal{J}}$ imply $a^{\mathcal{I}}=b^{\mathcal{J}}$.

The notion of consistency for a network of aligned ontologies can be naturally introduced thanks to the semantics of ontologies and alignments involved in the network.

Definition 5 (network consistency). Let $\left\langle\left\{O_{i}\right\}_{i=1}^{n},\left\{A_{i j}\right\}_{i, j=1, i \neq j}^{n}\right\rangle$ be a network of aligned ontologies in $\mathcal{A} \mathcal{L} C$. The network is consistent if there is a model $\mathcal{I}=\left\{\mathcal{I}_{i}\right\}_{i=1}^{n}$ of $O_{i}$ for all $1 \leq i \leq n$ such that

1. For each correspondence $a \approx b$ in $A_{i j}$ with $1 \leq i<$ $j \leq n, a^{I_{i}}=b^{I_{j}}$. For each correspondence $a \neq b$ in $A_{i j}$ with $1 \leq i<j \leq n, a^{\mathcal{I}_{i}} \neq b^{\mathcal{I}_{j}}$.

2. There are no pair of correspondences $a \approx b, a \neq b$ in $A_{i j}$. In this case, we say that $A_{i j}$ is clash-free.

3. For each correspondence $C \rightarrow D$ in $A_{i j}$ with $1 \leq i<$ $j \leq n$, if $D^{I_{j}}=\emptyset$ then $C^{I_{i}}=\emptyset$.

4. For each correspondence $\left\{\left\langle P_{k}, Q_{k}\right\rangle\right\}_{k=1}^{n}$ linkkey $\langle C, D\rangle$ in $A_{i j}$ with $1 \leq i<j \leq n$, if $\left(a_{k}^{i}\right)^{I_{i}}=\left(a_{k}^{j}\right)^{I_{j}}$, $\left\langle a^{I_{i}},\left(a_{k}^{i}\right)^{I_{i}}\right\rangle \in P_{k}^{I_{i}},\left\langle b^{I_{j}},\left(a_{k}^{j}\right)^{I_{j}}\right\rangle \in Q_{k}^{I_{j}}$ for all $1 \leq$ $k \leq n, a^{I_{i}} \in C^{I_{i}}, b^{I_{j}} \in D^{I_{j}}$ then $a^{I_{i}}=b^{I_{j}}$.

A network $N=\left\langle\left\{O_{i}\right\}_{i=1}^{n},\left\{A_{i j}\right\}_{i, j=1, i \neq j}^{n}\right\rangle$ entails a link key $\alpha$, written $N \vDash \alpha$, if every model of $N$ satisfies $\alpha$.

We finish this section by proving the following lemma which allows to reduce link key entailment to consistency of the network of aligned ontologies.

Lemma 1 (Reduction of link key entailment to consistency). Let $\left\langle\left\{O_{1}, O_{2}\right\}, A_{12}\right\rangle$ be a network of aligned ontologies in $\mathcal{A} \mathcal{L} C$. It holds that

$$
\begin{gathered}
\left\langle\left\{O_{1}, O_{2}\right\}, A_{12}\right\rangle \vDash\left(\left\{\left\langle P_{i}, Q_{i}\right\rangle\right\}_{i=1}^{m} \text { linkkey }\langle C, D\rangle\right) \text { iff } \\
\left\langle\left\{O_{1}^{\prime}, O_{2}^{\prime}\right\}, A_{12}^{\prime}\right\rangle \text { is inconsistent }
\end{gathered}
$$

with $O_{1}^{\prime}=O_{1} \cup\{C(x)\} \cup\left\{P_{i}\left(x, z_{i}\right)\right\}_{i=1}^{n}, O_{2}^{\prime}=O_{2} \cup\{D(y)\} \cup$ $\left\{Q_{i}\left(y, z_{i}^{\prime}\right)\right\}_{i=1}^{n}, A_{12}^{\prime}=A_{12} \cup\left\{z_{i} \approx z_{i}^{\prime}\right\}_{i=1}^{n} \cup\{x \nsim y\}, x, z_{1}, \cdots, z_{n}$ are new individuals in $O_{1}$ and $y, z_{1}^{\prime}, \cdots, z_{n}^{\prime}$ are new individuals in $\mathrm{O}_{2}$.

Proof. Let $\lambda=\left\{\left\langle P_{i}, Q_{i}\right\rangle\right\}_{i=1}^{n}$ linkkey $\langle C, D\rangle$. Assume that $\left\langle\left\{O_{1}, O_{2}\right\}, A_{12}\right\rangle \vDash \lambda$. Let us show that $\left\langle\left\{O_{1}^{\prime}, O_{2}^{\prime}\right\}, A_{12}^{\prime}\right\rangle$ is inconsistent. By contradiction, assume that $\left\langle\left\{O_{1}^{\prime}, O_{2}^{\prime}\right\}, A_{12}^{\prime}\right\rangle$ has a model $\mathcal{I}=\left\langle\mathcal{I}_{1}, \mathcal{I}_{2}\right\rangle$, i.e. $O_{1}^{\prime}$ and $O_{2}^{\prime}$ have models $\mathcal{I}_{1}$ and $I_{2}$ satisfying Definition 5. This implies that $I_{1}$ and
$I_{2}$ are models of $O_{1}$ and $O_{2}$. That means that $x^{I_{1}} \in C^{I_{1}}$, $y^{I_{2}} \in D^{I_{2}},\left\langle x^{I_{1}}, z_{i}^{I_{1}}\right\rangle \in P_{i}^{I_{1}},\left\langle y^{I_{2}}, z^{\prime I_{2}}\right\rangle \in Q_{i}^{I_{2}}, z_{i}^{I_{1}}=z_{i}^{\prime I_{2}}$ and $x^{I_{1}} \neq y^{I_{2}}$. This implies that $\mathcal{I} \not \lambda$. Thus, we have a model $\mathcal{I}$ of $\left\langle\left\{O_{1}^{\prime}, O_{2}^{\prime}\right\}, A_{12}^{\prime}\right\rangle$ such that $\mathcal{I} \not \forall \lambda$. Therefore, $\left\langle\left\{O_{1}^{\prime}, O_{2}^{\prime}\right\}, A_{12}^{\prime}\right\rangle \not \lambda$, which contradicts the assumption.

Assume now that $\left\langle\left\{O_{1}^{\prime}, O_{2}^{\prime}\right\}, A_{12}^{\prime}\right\rangle \not \forall \lambda$. Let us show that $\left\langle\left\{O_{1}^{\prime}, O_{2}^{\prime}\right\}, A_{12}^{\prime}\right\rangle$ is consistent. Since $\left\langle\left\{O_{1}^{\prime}, O_{2}^{\prime}\right\}, A_{12}^{\prime}\right\rangle \not \vDash \lambda$, then there exists an interpretation $\mathcal{I}=\left\langle\mathcal{I}_{1}, \mathcal{I}_{2}\right\rangle$ such that $\mathcal{I} \vDash\left\langle\left\{O_{1}^{\prime}, O_{2}^{\prime}\right\}, A_{12}^{\prime}\right\rangle$ and $\mathcal{I} \not \forall \lambda$.

Since $\mathcal{I} \not \lambda$, by the semantics of link keys, there exist $\delta, \delta_{1}, \ldots, \delta_{n} \in \Delta_{1}^{I_{1}}$ and $\delta^{\prime}, \delta_{1}^{\prime}, \ldots, \delta_{n}^{\prime} \in \Delta_{2}^{I_{2}}$ such that $\delta \in C^{I_{1}}, \delta^{\prime} \in D^{I_{2}},\left(\delta, \delta_{1}\right) \in P_{1}^{I_{1}},\left(\delta^{\prime}, \delta_{1}^{\prime}\right) \in Q_{1}^{I_{2}}, \ldots,\left(\delta, \delta_{n}\right) \in$ $P_{n}^{I_{1}},\left(\delta^{\prime}, \delta_{n}\right) \in Q_{n}^{I_{2}}, \delta_{1}=\delta_{1}^{\prime}, \ldots, \delta_{n}=\delta_{n}^{\prime}$ and $\delta \neq \delta^{\prime}$. Let us extend $\mathcal{I}$ by defining $x^{\mathcal{I}_{1}}=\delta, y^{\mathcal{I}_{2}}=\delta^{\prime}, z_{1}^{I_{1}}=$ $\delta_{1}, \ldots, z_{n}^{I_{1}}=\delta_{n}, z_{1}^{\prime I_{2}}=\delta_{1}^{\prime}, \ldots, z_{n}^{\prime I_{2}}=\delta_{n}^{\prime}$. Then, $\mathcal{I}$ is a model of $\left\langle\left\{O_{1}^{\prime}, O_{2}^{\prime}\right\}, A_{12}^{\prime}\right\rangle$. Therefore, $\left\langle\left\{O_{1}^{\prime}, O_{2}^{\prime}\right\}, A_{12}^{\prime}\right\rangle$ is consistent.

This lemma can be extended to a general network of aligned ontologies containing more than two ontologies.

\section{An algorithm for a network of aligned on- tologies}

The algorithm for deciding consistency of a network of aligned ontologies deals with pair by pair of ontologies in the network. For each pair of ontologies and an alignment between them, the algorithm repeats the following three tasks : propagating individual equalities from one ontology to the other via individual correspondences; applying link key correspondences which may lead to the addition of new individual correspondences; and propagating concept unsatisfiabilities from an ontology to the other via concept correspondences. The execution of a task may trigger the execution of another task. The execution of these tasks may lead to a change of ontologies and alignments in the network. The algorithm terminates on the pair of ontologies when the ontologies and the alignment reach stationarity. The first and second tasks are described in Algorithm 1 while the third one is outlined in Algorithm 2

The following lemma establishes that the propagation performed by Algorithms 1 and 2 and the consistency of the pair of the extended ontologies suffice to decide consistency of the network composed of the initial ontologies and the alignment.

Lemma 2 (reduction for a pair). Let $O_{1}, O_{2}$ be two consistent ontologies and $A_{12}$ be an alignment. We use $\widehat{O_{1}}$ and $\widehat{\mathrm{O}_{2}}$ to denote the resulting consistent ontologies obtained by calling propagatePair $\left(\mathrm{O}_{1}, \mathrm{O}_{2}, A_{12}\right)$. It holds that $\widehat{O_{1}}, \widehat{O_{2}}$ are consistent and $\widehat{A_{12}}$ is clash-free iff the network $\left\langle\left\{O_{1}, O_{2}\right\},\left\{A_{12}\right\}\right\rangle$ is consistent.

Before providing a complete proof of the lemma, we summarize the main arguments. The soundness of the if- 




direction of Lemma 2 is straightforward since Algorithms 1 and 2 add only logical consequences of the network to the ontologies and alignments. The soundness of the only-if-direction of the lemma is based on the following elements : (i) consistency of the extended ontologies and clash-freeness of the extended alignments imply consistency of the initial ontologies and clash-freeness of the initial alignements; (ii) Algorithms 1 and 2 make implicit all individual equalities, and thus potential clashes of the kind $a \approx b, a \approx b$ must be discovered. This ensures that two models of the extended ontologies satisfy individual correspondences; (iii) Algorithms 1 and 2 apply link keys until they are not applicable over the initial individuals in the ontologies. Since models of an $\mathcal{A} \mathcal{L} C$ ontology are treeshaped and $\mathcal{A} \mathcal{L} C$ does not allow for inverse roles, satisfaction of the link keys over the initial individuals is sufficient; and (iv) Algorithms 1 and 2 propagate concept unsatisfiabilities. If the "subsumer" of a concept correspondence is satisfiable then a model of the ontology can be extended such that the interpretation of the subsumer in this model is not empty. This implies that the concept correspondence is satisfied.

Proof. "If-direction". Assume that the network $\left\langle\left\{O_{1}, O_{2}\right\},\left\{A_{12}\right\}\right\rangle$ is consistent. By definition, $O_{i}$ has a model $I_{i}$ with $1 \leq i \leq 2$ such that they satisfy all correspondences $\alpha \in A_{12}$. We use $\widehat{A_{i j}}$ to denote the resulting alignment obtained by calling propagateEqualities $\left(O_{1}, O_{2}, A_{12}\right)$ and propagateUnsat $\left(O_{1}, O_{2}, A_{12}\right)$. We show that $I_{1}$ is a model of $\widehat{O_{1}}$. For this, we have to prove that :

- $\mathrm{a}_{0}^{I_{1}}=a_{n}^{I_{1}}$ if $a_{0} \approx a_{n}$ is added to $O_{1}$ by Line 9 in Algorithm 1 (this implies that there is no clash of the kind $a^{I_{1}}=b^{I_{1}}$, $\left.a^{I_{1}} \approx b^{I_{1}}\right)$. We have $a_{0} \approx a_{n}$ is added to $O_{1}$ if there is a sequence of equalities $a_{0} \approx a_{1}, \cdots, a_{n-1} \approx a_{n}$ such that $a_{i} \approx a_{i+1} \in \widehat{O_{1}} \cup \widehat{O_{2}} \cup \widehat{A_{12}}$ for $0 \leq i \leq n-1$. This sequence of equalities implies $a_{0}^{I_{1}}=a_{n}^{I_{1}}$. By using the same argument, we can show $a_{0}^{I_{2}}=a_{n}^{I_{2}}$ if $a_{0} \approx a_{n}$ is added to $O_{2}$ by Line 9 in Algorithm 1 . 


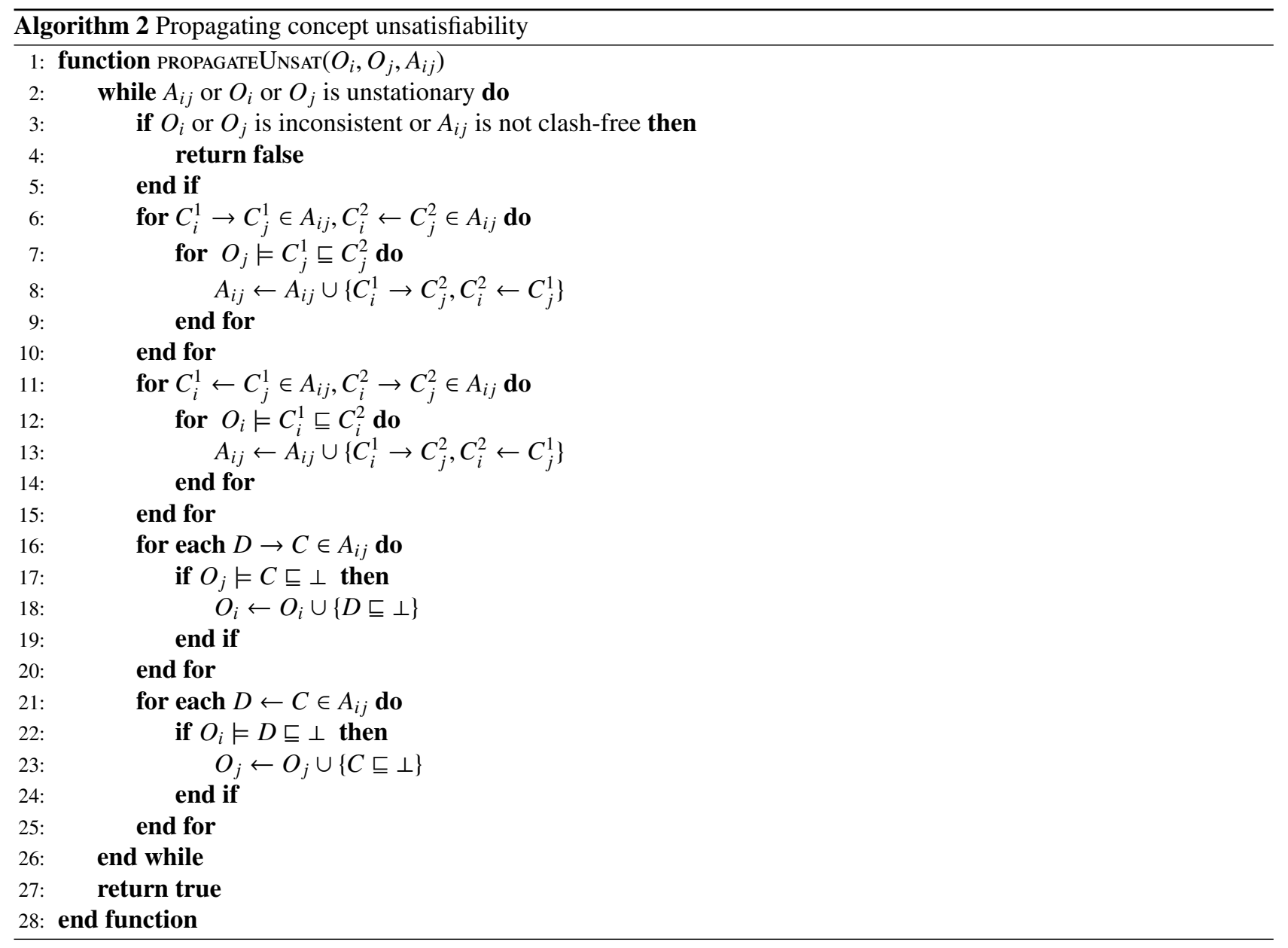

- $C_{0}^{I_{1}}=\emptyset$ if $C_{0} \sqsubseteq \perp$ is added to $O_{1}$ by Line 18 in Algorithm 2 (this implies that there is no clash of the kind $a^{I_{1}} \in C_{0}^{I_{1}}$, $C_{0}^{I_{1}}=\emptyset$ ). We have $C_{0} \sqsubseteq \perp$ is added to $O_{1}$ if there is a sequence $C_{0} \Rightarrow C_{1}, \cdots, C_{n-1} \Rightarrow C_{n}$ such that $\widehat{O_{1}} \vDash C_{i} \Rightarrow$ $C_{i+1}$ or $\widehat{O_{2}} \vDash C_{i} \Rightarrow C_{i+1}$ or $C_{i} \Rightarrow C_{i+1} \in \widehat{A_{12}}$ for $0 \leq i \leq$ $n-1$, and $\widehat{O}_{i} \vDash C_{n}^{\mathcal{I}_{i}} \sqsubseteq \perp(i \in\{1,2\})$ where " $\Rightarrow$ " represents " $\rightarrow$ " or " $\sqsubset$ " and $C \leftarrow D=D \Rightarrow C, C \sqsupseteq D=D \Rightarrow C$. This implies $C_{i}^{I_{i}}=\emptyset$ for $1 \leq i \leq n$. By using the same argument, we can show $C_{0}^{I_{2}}=\emptyset$ if $C_{0} \sqsubseteq \perp$ is added to $O_{2}$ by Line 23 in Algorithm 2

- The concepts $(C \sqcup \sim C)(a)$ and $(D \sqcup \sim D)(b)$ added by Lines 17 and 20 in Algorithm 2 do not change consistency since they are tautologies.

"Only-If-direction". Since $\widehat{O}_{i}$ is consistent, according to [7], $\widehat{O}_{i}$ has a tree-shaped model $\mathcal{I}_{i}$ where each interpretation domain $\Delta_{i}$ of $\mathcal{I}_{i}$ is composed of a set of initial individuals $I_{\text {old }}^{i}$ and a set of new individuals $I_{\text {new }}^{i}$ for $1 \leq i \leq 2$. Since $O_{i} \subseteq \widehat{O}_{i}, \mathcal{I}_{i}$ is a model of $O_{i}$ with $1 \leq i \leq 2$. We will extend $I_{1}$ and $I_{2}$ so that they satisfy the correspondences in $A_{12}$.

- For each $a \approx b \in \widehat{A_{12}}$, we define $a^{I_{1}}=a^{I_{2}}$. Thus, $a^{I_{1}}=$ $a^{I_{2}}$ for each $a \approx b \in A_{12}$ since $A_{12} \subseteq \widehat{A_{12}}$. By construction,
$I_{1}$ and $I_{2}$ satisfy all of the individual correspondences in $A_{12}$ according to Definition 4

- If $a \approx b \in \widehat{A_{12}}$ then $a \approx b \notin \widehat{A_{12}}$ since $\widehat{A_{12}}$ is clash-free.

- Let $C_{h} \rightarrow D_{h} \in A_{12}$. If $\widehat{O_{2}} \vDash D_{h} \sqsubseteq \perp$ then $C_{h} \sqsubseteq \perp$ is added to $\widehat{O_{1}}$ by Algorithm 2 Hence, $D_{h}^{I_{2}}=\emptyset$ implies $C_{h}^{I_{1}}=\emptyset$. Note that if $\widehat{O_{2}} \vDash D_{h} \sqsubseteq \perp$ then $\widehat{O_{2}^{\prime}} \vDash D_{h} \sqsubseteq \perp$ for all $\widehat{O_{2}} \subseteq \widehat{O_{2}^{\prime}}$.

Assume that $\widehat{O_{2}} \not \equiv D_{h} \sqsubseteq \perp$. Thus, $\widehat{O_{2}} \cup\left\{D_{h}\left(x_{h}\right)\right\}$ is consistent where $x_{h}$ is a new individual. According to [7], $\widehat{O_{2}} \cup\left\{D_{h}\left(x_{h}\right)\right\}$ has a tree-shaped model $I_{2}^{\prime}$ of $\widehat{O_{2}} \cup\left\{D_{h}\left(x_{h}\right)\right\}$. We show that if $\widehat{O_{2}} \cup\left\{D_{1}\left(x_{1}\right)\right\}$ and $\widehat{O_{2}} \cup$ $\left\{D_{2}\left(x_{2}\right)\right\}$ are consistent with new individuals $x_{1}, x_{2}$ then $\widehat{O_{2}} \cup\left\{D_{1}\left(x_{1}\right), D_{2}\left(x_{2}\right)\right\}$ is consistent. Indeed, running the standard tabaleau algorithm in [7] on $\widehat{O_{2}} \cup\left\{D_{1}\left(x_{1}\right)\right\}$ can build a set $\mathbf{T}$ of completion trees rooted at the initial individuals in $\widehat{O_{2}}$ and a completion tree $T_{x_{1}}$ rooted at $x_{1}$. Analogously, if the standard tableau algorithm runs on $\widehat{O_{2}} \cup\left\{D_{2}\left(x_{2}\right)\right\}$, it can build a set $\mathbf{T}$ ' of completion trees rooted at the initial individuals in $\widehat{O_{2}}$ and a completion tree $T_{x_{2}}$ rooted at $x_{2}$. All trees are clash-free and complete. Hence, the set of trees $\mathbf{T} \cup\left\{T_{x_{1}}, T_{x_{2}}\right\}$ would be built by the standard tableau algorithm when it runs on $\widehat{O_{2}} \cup\left\{D_{1}\left(x_{1}\right), D_{2}\left(x_{2}\right)\right\}$. 
Therefore, we can run the standard tableau algorithm in [7] on $\widehat{O_{2}} \cup\left\{D_{i}\left(x_{i}\right)\right\}_{i=1}^{m}$ to obtain a tree-shaped model $\mathcal{J}_{2}$ of $\widehat{O_{2}} \cup\left\{D_{i}\left(x_{i}\right)\right\}_{i=1}^{m}$ where $x_{h}$ is a new individual and $\widehat{O_{2}} \not=$ $D_{h} \sqsubseteq \perp$ for $1 \leq h \leq m$.

By using the same argument, we can obtain a treeshaped model $\mathcal{J}_{1}$ of $\widehat{O_{1}} \cup\left\{D_{1}^{\prime}\left(x_{1}^{\prime}\right), \cdots, D_{m^{\prime}}^{\prime}\left(x_{m^{\prime}}^{\prime}\right)\right\}$. By construction, $\mathcal{J}_{1}$ and $\mathcal{J}_{2}$ satisfy all of the concept correspondences in $A_{12}$ according to Definition 4 In addition, they remain to satisfy all of the individual correspondences in $A_{12}$. For the sake of the simplicity, we rename $\mathcal{J}_{1}$ and $\mathcal{J}_{2}$ to $\mathcal{I}_{1}$ and $\mathcal{I}_{2}$.

- Assume that $\left\{\left\langle P_{k}, Q_{k}\right\rangle\right\}_{k=1}^{n}$ linkkey $\langle C, D\rangle$ is a link key in $A_{12}$ and $\left(a_{k}^{1}\right)^{I_{1}}=\left(a_{k}^{2}\right)^{I_{2}},\left\langle a^{I_{1}},\left(a_{k}^{1}\right)^{I_{1}}\right\rangle \in P_{k}^{I_{1}},\left\langle b^{I_{2}},\left(a_{k}^{2}\right)^{I_{2}}\right\rangle \in$ $Q_{k}^{I_{2}}$ for all $1 \leq k \leq n, a^{I_{1}} \in C^{I_{1}}, b^{I_{2}} \in D^{I_{2}}$.

1. If $\left(a_{k}^{1}\right)^{I_{1}}=\left(a_{k}^{2}\right)^{I_{2}}$ then there is a sequence $a_{0} \approx$ $a_{1}, \cdots, a_{m-1} \approx a_{m}$ such that $a_{i} \approx a_{i+1} \in \widehat{O_{1}} \cup \widehat{O_{2}} \cup \widehat{A_{12}}$ for $0 \leq i \leq m-1$ with $a_{k}^{1}=a_{0}, a_{k}^{2}=a_{m}$. This implies that $a_{k}^{1} \approx a_{k}^{2} \in \widehat{A_{12}}$ for $1 \leq k \leq n$.

2. Since $\mathcal{I}_{1}$ and $\mathcal{I}_{2}$ are tree-shaped whose roots are the old individuals, the condition of the link key holds only if all individuals $a_{k}^{1}, a_{k}^{2}$ for $1 \leq k \leq n$, and $a, b$ are contained $I_{\text {old }}^{1} \cup I_{\text {old }}^{2}$. Hence, $\left\langle a^{I_{1}},\left(a_{k}^{1}\right)^{I_{1}}\right\rangle \in P_{k}^{I_{1}}$ iff $P_{k}\left(a^{\prime}, a_{k}^{\prime 1}\right) \in O_{1}$ with $O_{i} \vDash a \approx a^{\prime}, O_{i} \vDash a_{k}^{1} \approx a_{k}^{\prime 1}$ for $1 \leq k \leq n$ where $a, a^{\prime}$ and $a_{k}^{1}, a_{k}^{\prime 1}$ are old individuals.

3. Since $a^{I_{1}} \in C^{I_{1}}$ and $(C \sqcup \sim C)(a) \in O_{1}$ (Line 17, Algorithm 1 , we have $\widehat{O_{1}} \vDash C(a)$. Analogously, from $b^{I_{2}} \in D^{I_{2}}$ and $(D \sqcup \sim D)(b) \in O_{2}$ (Line 20. Algorithm 1), we obtain $\widehat{O_{2}}=D(b)$.

Therefore, the 3 items above trigger Line 26 in Algorithm 1 which adds to $\widehat{A_{12}}$ the assertion $a \approx b$. Thus, we obtain $a^{I_{1}} \approx b^{I_{2}}$.

We have proven that $I_{1}$ and $I_{2}$ are models of $O_{1}$ and $O_{2}$ which satisfy all of the correspondences in $A_{12}$.

We can observe that Algorithms 1 and 2 can be implemented in a decentralized manner since each call for checking ontology entailment or consistency can be sent to a local reasoner associated with the ontology located on a different site.

To check consistency of a network of aligned ontologies, it is needed to run Algorithms 1 and 2 on each pair of ontologies with the alignment between them until all ontologies and alignments are stationary. Note that saturating a pair of ontologies with the alignment can make a saturated pair of ontologies unsaturated. This is due to the fact that an ontology can be shared by several pairs of ontologies.

The following theorem is a consequence of Lemma 2

Theorem 1 (reduction for network). Let $\left\langle\left\{O_{i}\right\}_{i=1}^{n},\left\{A_{i j}\right\}_{i, j=1, i \neq j}^{n}\right\rangle$ be a network of aligned ontologies. We use $\widehat{O}_{i}(1 \leq i \leq n)$ to denote the resulting consistent ontologies obtained by calling
propagateOverNetwork $\left(\left\langle\left\{O_{i}\right\}_{i=1}^{n},\left\{A_{i j}\right\}_{i, j=1, i \neq j}^{n}\right\rangle\right)$. It holds that $\widehat{O}_{i}$ is consistent for all $1 \leq i \leq n$ and $\widehat{A_{i j}}$ is clash-free for all $1 \leq i<j \leq n$ iff the network $\left\langle\left\{O_{i}\right\}_{i=1}^{n},\left\{A_{i j}\right\}_{i, j=1, i \neq j}^{n}\right\rangle$ is consistent.

We now investigate the complexity of the algorithms. Under the hypothesis in which a call to reasoners associated with ontologies is considered as an oracle, i.e. an elementary operation, our algorithms are tractable.

Theorem 2. Let $\left\langle\left\{O_{i}\right\}_{i=1}^{n},\left\{A_{i j}\right\}_{i, j=1, i \neq j}^{n}\right\rangle$ be a network of aligned ontologies. The algorithm propagateOverNetwork $\left(\left\langle\left\{O_{i}\right\}_{i=1}^{n},\left\{A_{i j}\right\}_{i, j=1, i \neq j}^{n}\right\rangle\right)$ runs in polynomial time in the size of the network if each check of entailment or consistency occurring in the algorithms is considered as an oracle.

Proof. The complexity of propagateOverNetwork depends on the complexity of propagateEqual, propagateUnsat. When running these algorithms, each ontology can be monotonically extended. It is straightforward to obtain that the number of axioms of the form $C \sqsubseteq \perp$ added to ontologies $O_{i}$ and $O_{j}$ is bounded by a polynomial function in the size of initial alignments since $C$ must occur in an initial correspondence. Analogously, the number of individuals correspondences $a \approx b$ added to alignments $A_{i j}$ is bounded by a polynomial function in the size of initial alignments since $a, b$ must occur in an initial correspondence. This implies that the number of iterations of the while loops in Algorithms 1, 2 and 3 is bound by a polynomial function in the size of initial alignments.

In addition, the number of iterations of the for loops in Algorithms 1, 2 and 3 is bounded by a polynomial function in the size of initial alignments, the size of ontologies and the number of ontologies and alignments included in the network. This observation completes the proof.

\section{Examples}

This section provides some examples for showing how to use the algorithms presented in Section 4

Example 2. The ontologies and alignment in Example 1 can be rewritten as follows :

$O_{1}=\{D P \sqsubseteq P, D P(a)\}, O_{2}=\{P S \sqsubseteq R, R \sqsubseteq \neg D\}$,

$A_{12}=\{D P \rightarrow R, D P \rightarrow D,\langle p r, r e\rangle$ linkkey $\langle P, R\rangle\}$

If the correspondences are considered as standard subsumptions then the ontology $O_{1} \cup O_{2} \cup A_{12}$ is inconsistent. Indeed, assume that there is a model $\mathcal{I}=\left\langle\Delta^{\mathcal{I}},{ }^{I}\right\rangle$ of the ontology. This implies that $a^{I} \in D P^{I}, D P^{I} \subseteq R^{I}$ and $D P^{I} \subseteq D^{I}$. Thus, $a^{I} \in R^{I} \cap D^{I}$. However, we have $R^{I} \subseteq \Delta^{I} \backslash D^{I}$, which is a contradiction.

If we now interpret the correspondences under the semantics given in Definition 4 then there is no propagation needed according to Algorithms 1 and 2. It is obvious that 


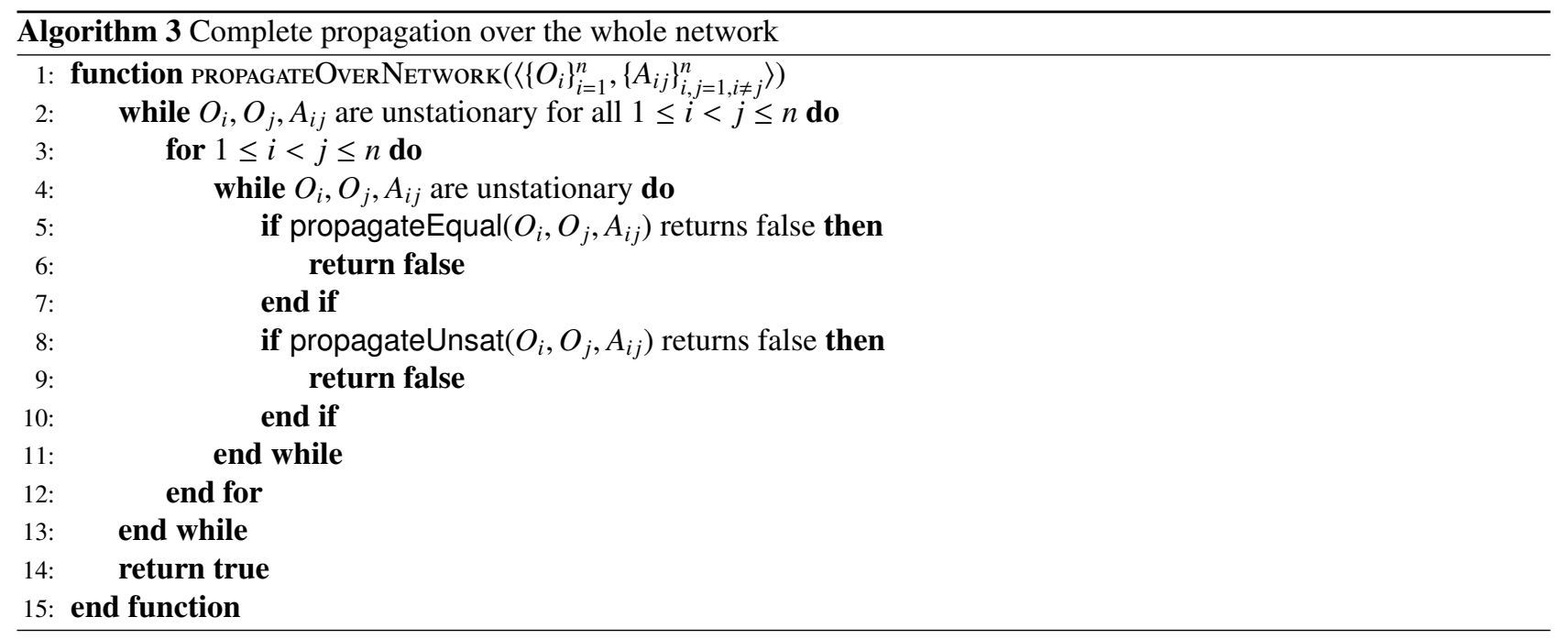

$O_{1}$ and $O_{2}$ are consistent, and the network $\left\langle\left\{O_{1}, O_{2}\right\}, A_{12}\right\rangle$ is consistent under the semantics given in Definition 4.

Example 3. In this example, we reduce two correspondences in Example 2 to one as follows.

$O_{1}=\{D P \sqsubseteq P, D P(a)\}, O_{2}=\{P S \sqsubseteq R, R \sqsubseteq \neg D\}$,

$A_{12}=\{D P \rightarrow R \sqcap D,\langle p r, r e\rangle$ linkkey $\langle P, R\rangle\}$

We now interpret the correspondence under the semantics given in Definition 4. Since $\mathrm{O}_{2} \vDash R \sqcap D \sqsubseteq \perp$, Algorithm 2 propagates unsatisfiability of $R \sqcap D$ to $O_{1}$ via the correspondence $D P \rightarrow R \sqcap D$. Hence, it adds $D P \sqsubseteq \perp$ to $O_{1}$. This leads to inconsistency of $\widehat{O_{1}}$. Therefore, the network $\left\langle\left\{O_{1}, O_{2}\right\}, A_{12}\right\rangle$ is not consistent.

Example 4. The ontologies and alignment in Example 1 can be rewritten as follows :

$O_{1}=\{D P \sqsubseteq P, D P(a)\}, O_{2}=\{P S \sqsubseteq R, R \sqsubseteq \neg D\}$,

$A_{12}=\{D P \rightarrow R, D P \rightarrow D,\langle p r, r e\rangle$ linkkey $\langle P, R\rangle\}$

We consider whether $\left\langle\left\{O_{1}, O_{2}\right\}, A_{12}\right\rangle \vDash \lambda$ where $\lambda=$ $\langle p r, r e\rangle$ linkkey $\langle P, R\rangle$. Due to Lemma 1$]$ we extend $O_{1}, O_{2}$ and $A_{12}$ by adding to $O_{1}$ assertions $D P(x), \operatorname{pr}\left(x, x_{1}\right)$, to $\mathrm{O}_{2}$ assertions $P S(y)$, re $\left(y, y_{1}\right)$, and to $A_{12}$ assertions $x_{1} \approx$ $y_{1}, x \neq y$. Let $\widehat{O_{1}}, \widehat{O_{2}}$ and $\widehat{A_{12}}$ be the extended ontologies and alignment. If there are models $\mathcal{I}_{1}$ and $\mathcal{I}_{2}$ of $\widehat{O_{1}}, \widehat{O_{2}}$ then, we have $x \in D P^{I_{1}}$ and $y \in P S^{I_{2}}$, and $D P^{I_{1}} \subseteq P^{I_{1}}$ and $P S^{I_{2}} \subseteq R^{I_{2}}$.Thus, the link key $\langle p r$, re $\rangle$ linkkey $\langle P, R\rangle$ is applicable, and Algorithm 1 adds $x \approx y$ to $\widehat{A_{12}}$. This leads to a clash in $\widehat{A_{12}}$ and thus the network $\left\langle\left\{\widehat{O_{1}}, \widehat{O_{2}}\right\}, \widehat{A_{12}}\right\rangle$ is not consistent. Therefore, $\left\langle\left\{O_{1}, O_{2}\right\}, A_{12}\right\rangle \vDash \lambda$ holds.

\section{Implementation and Experimental Results}

An implementation of the proposed algorithms has been integrated within a reasoner written in Java, called Draon [8], which already allowed to reason in a decentralized manner on a network of aligned ontologies under the IDDL semantics [14]. Algorithms 1, 2 and 3 can be naturally implemented such that reasoning tasks on ontologies can be independently performed by different reasoners located on different sites.

The architecture of Draon is despicted in Figure 1 A global reasoner implements Algorithm 3 This global reasoner loads alignments and executes Algorithm 3 It sends assertions/axioms different to local reasoners located on different sites. Then it asks local reasoners to check entailment and consistency of the ontology associated with each local reasoner. The global reasoner and each local reasoner use HermiT [11] as OWL reasoner. The communication between the global reasoner and all local reasoner is based on OWLLink [9]. When connecting to a local reasoner, the global reasoner creates a Java thread which deals with the communication between them. Data shared by the threads are synchronized and protected by using semaphores. Note that we can replace HermiT with any OWL reasoner since OWLLink supports a generic OWL reasoner.

Table 1 provides information on the ontologies and alignments used for the experiments. These datasets are taken from OAEI2012 $2^{2}$ and OAEI2018 ${ }^{3}$ Campaigns. We have chosen small ontologies and alignments such as iasted.owl, sigkdd.owl, iasted-sigkdd.rdf to test our algorithm on alignments with link keys since they are well understood and manually checkable. This allows us to create manually relevant link keys (to our best knowledge, there is no system which can generate link keys expressed in the alignment syntax). In addition, we have selected large ontologies and alignment such as SNOMED, FMA, FMA-SNOMED in order that the difference between the reasoning complexities of the two semantics IDDL (implemented in Draon) and APPROX (the new semantics pre-

2. cs.ox.ac.uk/isg/projects/SEALS/oaei/2012/

3. oaei.ontologymatching.org/2018/conference 


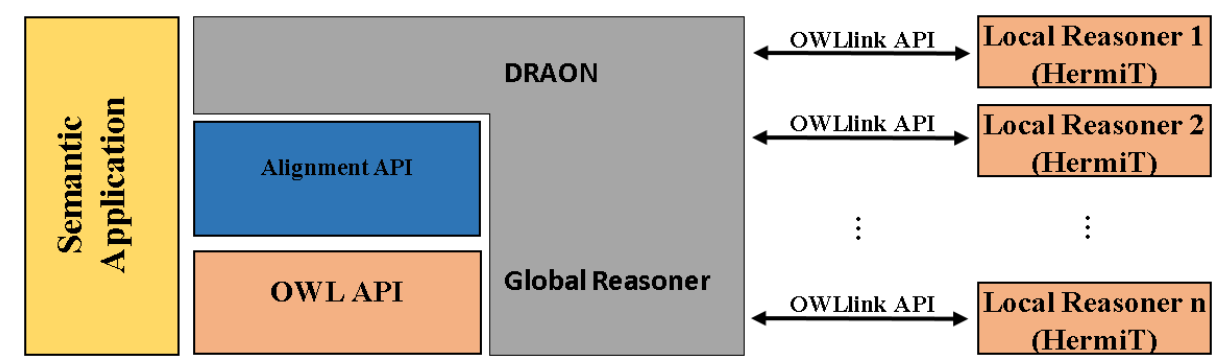

Figure 1 - Architecture of Draon

\begin{tabular}{|c|c|c|c|c|}
\hline & Concepts & Roles & Individuals & Axioms/Correspondences \\
\hline Iasted & 141 & 38 & 6 & 551 \\
\hline Sigkdd & 50 & 18 & 5 & 210 \\
\hline iast-sigkdd (without link keys) & & & & 15 \\
\hline Conference & 60 & 46 & 2 & 414 \\
\hline Ekaw & 74 & 33 & 4 & 351 \\
\hline conference-ekaw (without link keys) & & & & 27 \\
\hline Cmt & 30 & 49 & 3 & 327 \\
\hline Edas & 104 & 30 & 117 & 1025 \\
\hline cmt-edas (without link keys) & & & & 47467 \\
\hline FMA & 10157 & 0 & 0 & 47104 \\
\hline SNOMED & 13412 & 18 & 0 & 9139 \\
\hline FMA-SNOMED (without link keys) & & & & 135556 \\
\hline NCI & 25591 & 87 & 0 & 3038 \\
\hline FMA-NCI (without link keys) & & & & \\
\hline
\end{tabular}

TABLE 1 - Ontologies and aligments without link keys and their characteristics

\begin{tabular}{|c|c|c|c|c|}
\hline Ontology 1 & Ontology 2 & Alignment & IDDL & APPROX \\
\hline Iasted & Sigkdd & iasted-sigkdd (without link keys) & $3.5 \mathrm{~s}$ & $9 \mathrm{~ms}$ \\
\hline Conference & Ekaw & conference-ekaw (without link keys) & $7.5 \mathrm{~s}$ & $11 \mathrm{~ms}$ \\
\hline Cmt & Edas & cmt-edas (without link keys) & $7.5 \mathrm{~s}$ & $16 \mathrm{~ms}$ \\
\hline FMA & SNOMED & FMA-SNOMED (without link keys) & $>15$ minutes & $81 \mathrm{~s}$ \\
\hline FMA & NCI & FMA-NCI (without link keys) & $>15$ minutes & $10 \mathrm{~s}$ \\
\hline
\end{tabular}

TABLE 2 - Execution time for checking consistency of ontology networks according to different semantics

\begin{tabular}{|c|c|c|c|}
\hline Ontology 1 & Ontology 2 & Alignment & Consistency in APPROX \\
\hline Iasted & Sigkdd & iast-sigkdd (with link keys) & $9 \mathrm{~ms}$ \\
\hline Conference & Ekaw & conference-ekaw (with link keys) & $11 \mathrm{~ms}$ \\
\hline Cmt & Edas & cmt-edas (with link keys) & $17 \mathrm{~ms}$ \\
\hline
\end{tabular}

TABLE 3 - Execution time (in milliseconds) for checking consistency of ontology networks with link keys

sented in the paper) is more noticeable.

We use two remote DELL servers with Intel $3.4 \mathrm{GHz}$ Processor 8 cores and 32Gb RAM on which two HermiTbased local reasoners are running. The global reasoner is also launched on a third computer with the same configuration.

We run Draon to check consistency of several networks of ontologies each of which is composed of ontologies and alignment described in Table 1 The results are put in Table 2 which shows execution times of Draon under the two different semantics IDDL and APPROX. The difference of the performances in time results from the fact that reasoning under IDDL may require in the worst case an exponential number of message exchanges between the global reasoner 
and the local reasoners while reasoning under APPROX needs at most a polynomial number of message exchanges.

Table 3 provides first experimental results when running Draon to check consistency of networks containing small ontologies and alignment with link keys. The alignments in this table are obtained by adding to the corresponding alignments in Table 2 some link keys manually created.

\section{Conclusion and Future Work}

We have presented a new semantic of alignments which is weaker than the standard semantics. This weakened semantics of alignments allows us to express correspondences between ontologies of different nature on the one hand and to propose an efficient algorithm for reasoning on a network of ontologies with alignments containing link keys on the other hand. This new kind of correspondences is useful for establishing data links between heterogeneous datasets. The complexity of the proposed algorithm is polynomial in the size of the network if each call for checking ontology entailment or consistency is considered as an oracle. We have integrated an implementation of our algorithm within a distributed reasoner, called Draon, and reported some experimental results.

Our algorithm can be extended to deal with ontologies expressed in a more expressive Description Logic than $\mathcal{A} \mathcal{L} C$ in condition that the new logic does not allow for inverse roles. This restriction on expressiveness prevents the current algorithm from merging individuals which are initially not in the ontology. Another extension of the current work aims at adding role correspondences to alignments. This may require the algorithm to support ontologies allowing for hierarchy of roles and the negation of roles. We plan to carry out experiments of Draon on ontologies and alignments located on a large number of nodes equipped with a local reasoner. New evaluations of Draon on alignments with a large number of link keys are also expected.

\section{Acknowledgements}

This work has been partially supported by the ANR project Elker (ANR-17-CE23-0007-01).

\section{Références}

[1] Adjiman, Philippe, Philippe Chatalic, François Goasdoué, Marie-Christine Rousset et Laurent Simon: Distributed Reasoning in a Peer-to-Peer Setting : Application to the Semantic Web. J. Artif. Intell. Res., 25 :269-314, 2006.

[2] Atencia, Manuel, Jérôme David et Jérôme Euzenat: Data interlinking through robust linkkey extraction.
Dans Proc. 21st european conference on artificial intelligence (ECAI), pages 15-20. IOS press, 2014.

[3] Bao, Jie, Doina Caragea et Vasant G Honavar: A distributed Tableau Algorithm for Package-based Description Logics. Dans Proceedings of the ECAI Workshop on Context Representation and Reasoning, 2006.

[4] Borgida, Alex et Luciano Serafini: Distributed Description Logics : Assimilating information from peer sources. Journal Of Data Semantics, (1) :153-184, 2003.

[5] Gmati, Maroua, Manuel Atencia et Jérôme Euzenat: Tableau extensions for reasoning with link keys. Dans Proceedings of the 11th International Workshop on Ontology Matching, pages 37-48, 2016.

[6] Grau, Bernardo Cuenca, Bijan Parsia et Evren Sirin: Combining OWL Ontologies Using E-connections. Journal Of Web Semantics, 4(1), 2006.

[7] Horrocks, Ian, Ulrike Sattler et Stephan Tobies: Reasoning with Individuals for the Description Logic SHIQ. Dans Proc. of the 17th Int. Conf. on Automated Deduction (CADE 2000), pages 482-496. Springer, 2000.

[8] Le Duc, Chan, Myriam Lamolle, Antoine Zimmermann et Olivier Curé: DRAOn : A Distributed Reasoner for Aligned Ontologies. Dans Informal Proceedings of the 2nd International Workshop on OWL Reasoner Evaluation (ORE-2013), pages 8186, 2013.

[9] Liebig, Thorsten, Marko Luther, Olaf Noppens et Michael Wessel: OWLlink. Semantic Web, 2(1) :23-32, 2011.

[10] Serafini, Luciano et Andrei Tamilin: DRAGO : Distributed Reasoning Architecture for the Semantic Web. Dans Proceedings of the Europeen Semantic Web Conference, pages 361-376, 2005.

[11] Shearer, Rob, Boris Motik et Ian Horrocks: HermiT: A Highly-Efficient OWL Reasoner. Dans Proc. of the 5th Int. Workshop on OWL : Experiences and Directions (OWLED 2008 EU), 2008.

[12] Sirin, Evren, Bijan Parsia, Bernardo Cuenca Grau, Aditya Kalyanpur et Yarden Katz: Pellet : a pratical OWL-DL reasoner. Journal of Web Semantics, 5(2) :51-53, 2007.

[13] Zimmermann, Antoine et Jérôme Euzenat: Three semantics for distributed systems and their relations with alignment composition. Dans Proc. 5th International Semantic Web Conference (ISWC), pages 1629, 2006.

[14] Zimmermann, Antoine et Chan Le Duc: Reasoning with a Network of Aligned Ontologies. Dans RR, pages 43-57, 2008. 